\title{
HIGROMA QUIISTICO DEL CUELLO. INFORME DE UN CASO
}

CYSTIC NECK HYGROMA. REPORT OF A CASE

HIGROMA QUÍSTICO DE PESCOÇO. INFORME DUM CASO

\author{
JAMES NEIRA BORJA',2, JHON CORONEL RUILOVA2², DIEGO PAREDES CALAHORRANO², MARía dE FÁtIMA NEIRA VERDUGA ${ }^{3}$ \\ 1 Universidad de Guayaquil, Guayaquil, Ecuador \\ 2 Hospital Luis Vernaza, Guayaquil, Ecuador \\ ${ }^{3}$ Omni hospital, Guayaquil, Ecuador
}

Resumen

Los linfangiomas son malformaciones congénitas del sistema linfático relativamente infrecuentes que normalmente se presentan en los primeros años de la infancia; constituyen la causa más frecuente de tumoración de la región lateral del cuello presentes al nacimiento. Un síntoma común es una tumoración en el cuello que se observa ya sea en el momento de nacer o más tarde, después de una infección respiratoria. La presentación en la edad adulta es rara y su tratamiento es normalmente quirúrgico. Se presenta caso de paciente masculino de 50 años de edad, con cuadro clínico de masa tumoral cervical izquierda de 1 año de evolución, sin relación a traumatismos previos, la que generaba dificultad respiratoria por su tamaño y compresión de estructuras cercanas como la vía aérea, además de dificultad para la deglución, luego de los exámenes de imagen se evidencia en ecografía una tumoración quística de 12 x 10cm; así mismo se practica una punción por aspiración por aguja fina, la cual no reportó signos de malignidad al estudio citológico, por lo que es intervenido quirúrgicamente, realizándose la extirpación completa del tumor; y, preservando estructuras vasculares, nerviosas y glandulares. Su estudio anatomopatológico confirmó el diagnóstico de Linfangioma Quístico. Luego del alta médica acude a controles periódicos por consulta externa sin evidenciarse recidivas, concluyendo que su diagnóstico y manejo quirúrgico fue el correcto, a pesar de su gran tamaño y el tiempo de evolución.

PALABRAS CLAVE: linfangioma quístico, anomalías linfáticas, neoplasias de cabeza y cuello.

Abstract

Lymphangiomas are relatively infrequent congenital malformations of the lymphatic system that usually occur in the early years of childhood; they constitute the most frequent cause of tumoration of the lateral neck region present at birth. A common symptom is a tumor in the neck that is observed either at birth or later, after respiratory infection. Its presence in adulthood is rare and its treatment is usually surgical. The case of a 50-year-old male patient with clinical picture of left cervical tumor mass of 1 year of evolution is presented, without relation to previous traumas, which generated respiratory difficulty due to size and compression of structures such as the airway; in addition to difficulty in swallowing, after imaging tests, a cystic tumor of $12 \mathrm{x}$ $10 \mathrm{~cm}$ was evidenced through ultrasound; likewise, fine needle aspiration was performed, which did not show signs of malignancy in the cytological study, so it was surgically treated through complete removal of the tumor; and, preserving vascular, nervous and glandular structures. The anatomopathological study confirmed diagnosis of Cystic Lymphangioma. After medical discharge, he went to periodic check-ups at outpatient consultation without evidence of recurrence, concluding that diagnosis and surgical management were correct, despite large size of tumor and evolution time.

KEYWORDS: lymphangioma cystic, lymphatic abnormalities, head and neck neoplasms.

Resumo

Os linfangiomas são malformações congénitas do sistema linfático relativamente infrequentes que normalmente se apresentam nos primeiros anos da infância; constituem a causa mais frequente de tumoração da região lateral do pescoço presentes ao nascimento. Um sintoma comum é uma tumoração no pescoço que se observa num momento do nascimento ou mais tarde, depois de uma infeção respiratória. A apresentação na idade adulta é rara e 0 seu tratamento e normalmente cirúrgico. Se apresenta um caso de um paciente masculino de 50 anos de idade, com quadro clínico de massa tumoral cervical esquerda de 1 ano de evolução, sem relação a traumatismos prévios, a que gerava dificuldade respiratória pelo seu tamanho e comprometimento das estruturas próximas como as vias aéreas, ademas, de dificuldade para engolir, logo dos exames de imagem se observa em ecografia uma tumoração quística de $12 \times 10 \mathrm{~cm}$. Também, uma punção lombar com aspiração por agulha fina foi praticada, a qual não reportou sinais de malignidade ao estudo citológico, e por isso foi feita intervenção cirúrgica, sendo feita a extirpação completa do tumor; e preservando estruturas vasculares, nervosas e glandulares. 0 seu estudo anatomopatológico confirmou o diagnostico de Linfangioma Quistico. Logo da alta medica, realiza controles periódicos pela consulta externa sem evidencia de recidivas, finalizando que o seu diagnostico e manejo cirúrgico foi o correto, a pesar de seu grande tamanho e tempo de evolução.

PALABRAS-CHAVE: linfangioma cístico, anormalidades linfáticas, neoplasias de cabeça e pescoço. 
INTRODUCCIÓN

Es un tumor del sistema linfático de origen embrionario originado por obstrucción del drenaje de los sacos linfáticos. Se asocia a obstrucción linfática generalizada, pudiendo presentar derrame pleural, ascitis y edema cutáneo ${ }^{1}$. Área posterolateral del cuello, el congénito infantil constituye la variante más frecuente. Su patogenia es desconocida, ya que la embriogénesis del sistema linfático no se ha esclarecido de forma completa todavía. ${ }^{2}$ La aparición en edad adulta es muy poco frecuente y se deben descartar los de causa adquirida o iatrogénica. Se presentan en una relación de 2:1 en el lado izquierdo con respecto al derecho del cuello, probablemente porque el conducto torácico ingresa a la vena subclavia por la izquierda, su crecimiento va de gradual a rápido con compresión o desplazamiento de estructuras adyacentes. ${ }^{3}$ Los higromas aparecen como masas quísticas de consistencia blanda, móvil, no sensible, única o múltiple. ${ }^{4}$ Se ubican preferentemente en el triángulo posterior del cuello, su tamaño puede variar desde algunos milímetros a varios centímetros de diámetro, pueden ser indoloras, mal delimitadas, fluctuantes, lobuladas, multitabicadas. ${ }^{5}$ Cuando se infectan se presentan con signos inflamatorios, en general los síntomas están relacionados con la localización del quiste y su tamaño. Si la masa comprime la vía aérea ocasiona síndrome de dificultad respiratoria y trastornos de la deglución, cuando está localizado en la región perifaríngea puede ocasionar alteraciones en la función de la articulación temporomandibular. ${ }^{6}$ Las complicaciones suelen ser menos graves y la cirugía menos dificultosa que en el niño.

Tienden a desarrollarse en íntimo contacto con grandes venas y conductos linfáticos. ${ }^{7}$

TABLA 1. LOCALIZACIÓN MÁS FRECUENTE DE LOS HIGROMAS QUÍSTICOS

\begin{tabular}{ll}
\hline HIGROMA Quístico \\
\hline $75 \%$ & Región lateral cuello detrás del músculo esternocleidomastoideo. \\
\hline $20 \%$ & Región submaxilar o Región subglótica. \\
\hline $5 \%$ & Axila, Mediastino, Retroperiotoneo, Pelvis. \\
\hline
\end{tabular}

CASO CLÍNICO

Paciente masculino, 50 años de edad, mestizo, agricultor, que ingresa al hospital por presentar tumoración blanda localizada en región cervical izquierda, única de 6 meses de evolución, sin relación a traumatismos previos que apareció sin causa aparente y que fue creciendo progresivamente. Jamás ha sido valorada anteriormente.
Al realizar el examen físico el tamaño de la tumoración era aproximadamente $15 \mathrm{~cm}$ de diámetro ubicado en el triángulo izquierdo posterior del cuello, llama la atención la dificultad respiratoria por compresión de la vía aérea y dificultad para la deglución, motivos por los que acude a nuestra institución hospitalaria.

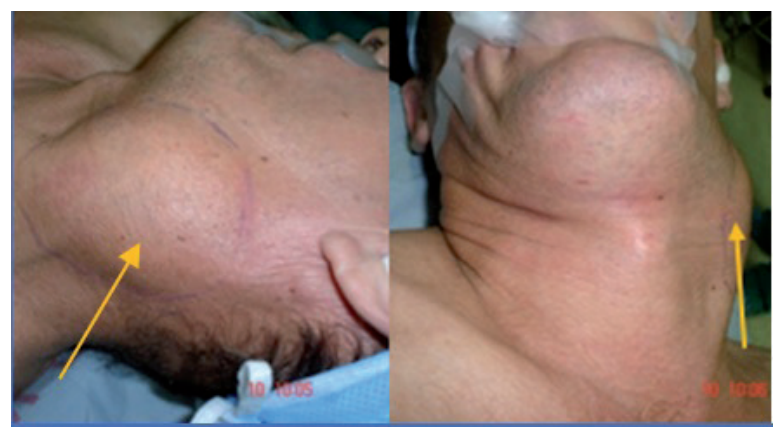

Figura 1. Paciente presentando masa tumoral en región cervical izquierda. Izquierda: vista lateral. Derecha: vista anteroposterior.

A la palpación se apreciaba consistencia blanda, móvil no adherida a planos profundos. Como antecedentes personales presentaba hipertensión arterial y dislipidemia, las mismas que se encontraban en tratamiento. Se realizó ecografía de partes blandas de cuello en donde se evidencia una tumoración quística de límites definidos con diámetros D1: $12 \mathrm{~cm} \mathrm{x} \mathrm{D2:} 10 \mathrm{~cm}$.

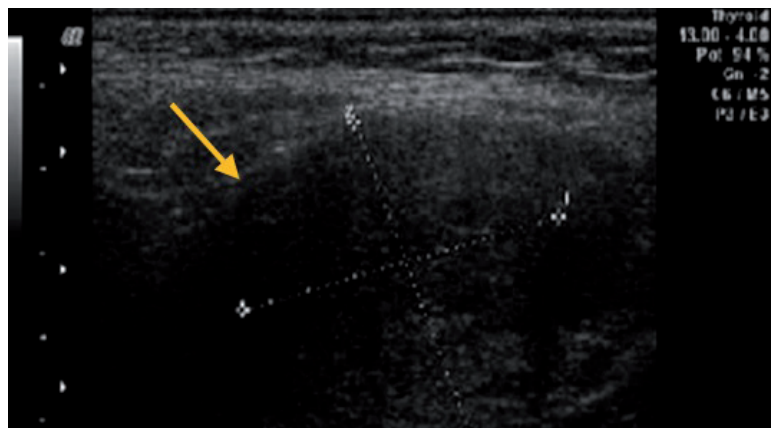

Figura 2. Se evidencia imagen hipoecogénica, con refuerzo posterior, que manifiesta contenido líquido, de gran tamaño, que desplaza estructuras cercanas.

Solicitamos una punción aspiración por agua fina (PAAF) en la cual se extraen 20cc de líquido amarillento sin criterios citológicos concluyentes de malignidad.

Durante su hospitalización en sala San Aurelio persiste la dificultad respiratoria por lo que se decide intervenir quirúrgicamente, realizándose una cervicotomía lateral izquierda ampliada para acceder al espacio cervical posterior en donde se observa la gran tumoración; se consigue la extirpación completa del tumor con preservación de estructuras vasculares, nerviosas y glandulares del cuello más drenaje tubular externo. 


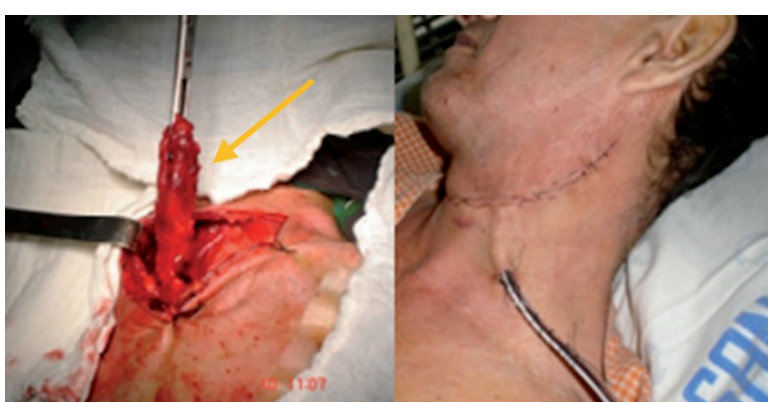

Figura 3. Cervicotomía lateral izquierda ampliada más drenaje de herida quirúrgica por contrabertura.

El informe anatomopatológico confirmó el diagnóstico de linfangioma quístico.

El paciente no presentó complicaciones post quirúrgicas y acude a consultas para revisiones periódicas, que fueron cada mes por 1 ocasión durante 6 meses consecutivos.

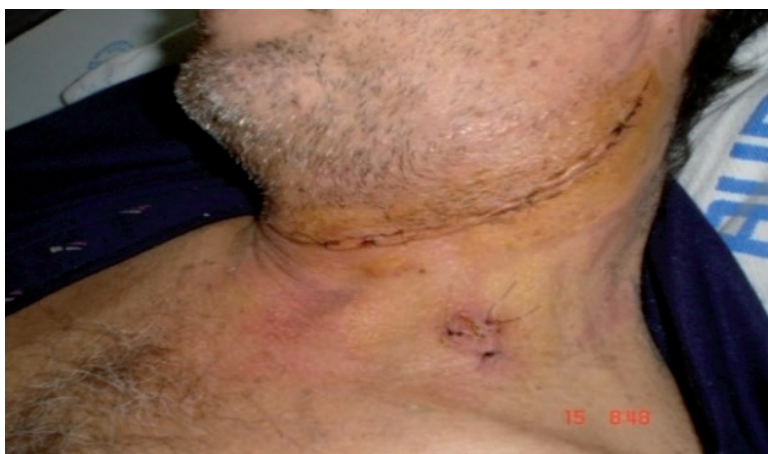

Figura 4. Paciente al quinto día de postoperatorio luego de que se ha retirado drenaje cervical.

DISCUSIÓN

Las masas quísticas cervicales congénitas son un grupo de lesiones infrecuentes usualmente diagnosticadas en la infancia. De ellas el higroma quístico es una de las más frecuentes, siendo la forma más común de linfangiomal. Es una anomalía de los vasos linfáticos yugulares que impide el correcto drenaje de la linfa, mayormente en el área de la cabeza o cuello y a menudo aparecen como una protrusión blanda debajo de la piel ${ }^{8}$, así pues el Linfangioma cervical posterior es una malformación congénita del sistema linfático compuesta de quistes llenos de linfa y en ocasiones de sangre, limitados por endotelio vascular y tejido conjuntivo. ${ }^{9}$ La incidencia global calculada por Filston en un estudio fue de 1.2 -2.8 casos por $1000^{10}$. Pueden aparecer a cualquier edad aunque el $50 \%$ son visibles al nacimiento y un $90 \%$ antes de los dos años de vida. La localización más frecuente es en triángulo posterior del cuello $75 \%$ de los casos), como en el caso de nuestro paciente; $20 \%$ en el triángulo submandibular, y $5 \%$ puede presentarse en abdomen, retroperitoneo, mediastino, e incluso en múltiples localizaciones ${ }^{11}$. Otras localizaciones excepcionales fuera del área de cabeza y cuello son: inguinal, axila, nuca y mediastino. ${ }^{12}$ No existen factores de riesgo relacionados con el sexo, origen étnico ni predominio en ninguno de los lados pero sí se han asociado a anomalías cromosómicas como el síndrome de Turner (la más frecuente), varias trisomías, síndromes de Klinefelter y Noonan. ${ }^{13}$

Se describen tres tipos de linfangiomas según el tamaño de los quistes ${ }^{14}:$ a) Capilar, menores de 1 $\mathrm{mm}, \mathrm{b}$ ) Cavernoso o micro quístico, menores de $5 \mathrm{~mm}$. c) Linfangioma macroscópico o higroma quístico, mayores de $10 \mathrm{~mm}$, por lo tanto sería este el que encaje en la descripción de este caso clínico quirúrgico. La aparición en edad adulta es muy poco frecuente y para algunos autores se debe a una confluencia de factores predisponentes (áreas con vasos linfáticos anómalos) y precipitantes (traumatismos en la zona o iatrogenia) ${ }^{15}$, aunque en nuestro caso no se identifique la causa, asumimos que probablemente se deba a una anomalía congénita. El diagnóstico es fundamentalmente clínico. El estudio de diagnóstico de elección es la tomografía computada (TAC), que permite su evaluación y que además permite seleccionar el abordaje quirúrgico. Sin embargo en nuestro hospital por motivos técnicos no se disponía durante ese período de la TAC, para ello decidimos realizar el ECO de cuello y partes blandas el cual mostró un tumor hipoecoico, con zona de refuerzo, sin trabéculas y que mostraba desplazamiento de estructuras adyacentes ${ }^{11}$, como apreciamos en la figura 2. Su resección completa continúa siendo el tratamiento de elección principalmente en tumoraciones loculadas. ${ }^{16}$

El diagnóstico diferencial se debe realizar principalmente con otras malformaciones vasculares como el hemangioma cavernoso, que se compone de paredes gruesas y contenido puramente hemático. La extirpación quirúrgica de los linfangiomas en edad adulta como en nuestro caso, es menos dificultosa que el niño y las recidivas son raras. El tratamiento de elección para los linfangiomas macroquísticos limitados al cuello es la extirpación quirúrgica aunque muchos autores consideran adecuada la abstención terapéutica en los casos asintomáticos, ya que la evolución natural de los linfangiomas no es bien conocida y se ha descrito involución espontánea completa en muchos de casos de linfangiomas 
bien delimitados. ${ }^{1,7,17}$ Otros autores prefieren una cirugía precoz antes de que los imprevisibles brotes inflamatorios causen complicaciones y dificulten la posterior disección, motivo por el cual nosotros decidimos previo a completar exámenes de imagen, laboratorio y citológicos una intervención quirúrgica temprana.

Otros métodos terapéuticos alternativos o añadidos a la cirugía que se han utilizado son la escleroterapia, la inyección intraquística de OK432, radiofrecuencia, interferón alfa y ciclofosfamida. Algunos de ellos como la escleroterapia con solución alcohólica de prolamina se han usado como técnica de elección en algunos casos. La recidiva es frecuente con cualquier técnica sobre todo dentro del primer año tras la extirpación. La mortalidad varía entre 0 y $6 \%$ sobre todo por las complicaciones que se produce al presionar la vía aérea. ${ }^{18}$

CONCLUSIONES

La identificación básicamente es clínica, pero no se puede dejar de respetar el criterio patológico, su detección rápida ayuda mucho en la disminución de complicaciones que tienen que ver con la compresión mecánica que este puede ejercer sobre estructuras cercanas. Deberá intentarse siempre la incisión que permita la adecuada exposición del linfangioma y de las estructuras adyacentes y que, además, ofrezca un adecuado resultado estético. Aunque inusual en la edad adulta, debe formar parte del diagnóstico diferencial de los tumores cervicales. Su tratamiento es quirúrgico pero puede intentarse la escleroterapia como tratamiento alternativo. ${ }^{19}$

CONFLICTO DE INTERESES

Los autores declaran no tener conflicto de interés.

\section{REFERENCIAS BIBLIOGRÁFICAS}

1. John R. Mernagh, Patrick T. Mohide, Raymond E. Lappalainen, and James G. Fedoryshin. US Assessment of the Fetal Head and Neck: A State-of-the-Art Pictorial Review. October 1999 RadioGraphics, 19, S229-S241.

2. Briceño L. y Calcaño G. Concejo de desarrollo Científico y Humanístico: Cirugía Pediátrica en Universidad Central de Venezuela Volumen II, 2003; 1308.

3. Elsevier Los 100 diagnósticos principales en cabeza y cuello, edición en español, primera edición, Génova $2006 ; 332$.
4. Uribe F., Arang M., Cirugía Pediátrica, Editorial Universidad de Antioquia, 1era Edición Julio 2006; 594.

5. Bracher, Frontier, Semiología Quirúrgica: Antología de Experiencias, textos y casos.- Santo Domingo: Instituto tecnológico de Santo Domingo, 2007; 97.

6. Wittekindt C, Michel O, Streeppel M, Roth B, Quante G, Beutner D, Guntinas-Lichius O. Lymphatic Malfornations of the head and neck: Introduction of a disease score for chindren, Cologne Disease Score (CDS). Int J of Ped Otorhinolaryngology. 2006; 70: 1205-1212.

7. Pera C. Cirugía, Fundamentos, indicaciones y opciones técnicas. Tomo II/2. MASSON S.A 1996; 52.

8. Hübner M., Malformaciones congénitas: Diagnóstico y Manejo neonatal Editorial Universitaria, 2005; 97-99.

9. Brito C. Azevedo C. Rossi J. Cirugía Vascular, Angiología 2da Ed 2004; 26:527; 32:633.

10. Karlos PD, Spencer MG, Lee M, Hamid BN. Cervical cystic hygroma/lymphangioma: an acquired idiopathic late presentation.J Laryngol Otol 2005 Jul;119(7):561-3.

11. P.M. Rodríguez, J.R. Navarro, Higroma quístico, An Orl Mex, 54 (1) (2009), pp. 28-31.

12. Fitzgerald-Kaufer-Malkani. Ortopedia. Tomo II, Editorial Panamericana. Edición 2002; 1192.

13. Pinto P, Malformaciones Congénitas, Ediciones Uninorte 1998; 125.

14. Revista ELSEVIER Valenzuela, M. José; Santero, M. Pilar; Arribas, M. Dolores; Córdoba, Elena; Martínez, Fernando, Linfangioma quístico cervical en el adulto Publicado en Cir Esp. 2010; 87:122-3. - vol.87 núm 02

15. Cabrerizo-Merino MC, Oñate Sánchez R, Romero Maroto M. El Hemangiolinfangioma en la infancia: localización inusual. RCOE 2004;9(1):89-92

16. Y. Guruprasad, D.S. Chauhan, Cervical cystic higroma, J Maxillofac Oral Surg, 11 (3) (2012), pp. 333-336

17. Kennedy TL, Whitaker M, Pellitteri P, Wood WE. Cystic hygroma/lymphangioma: a rational approach to management.Laryngoscope 2001;111 (11 Pt 1): 1929-37.

18. Briceño L., Calcaño G. Concejo de desarrollo Científico y Humanístico: Cirugía Pediátrica en Universidad Central de Venezuela Volumen II, 2003; 1309.

19. L. Vaid, M. Gupta, N. Gupta, P.P. Singh, Bleomycin sclerotherapy in a rare case of adult-onset cervical lymphangioma, Ear Nose Throat J, 89 (1) (2010), pp. E23-E26 PMid:20155685 